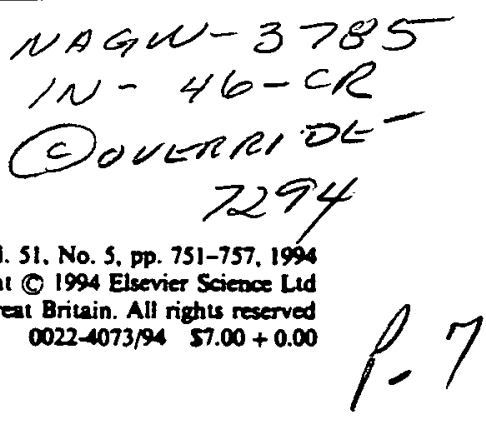

\title{
THE DETAILED BALANCE REQUIREMENT AND GENERAL EMPIRICAL FORMALISMS FOR CONTINUUM ABSORPTION
}

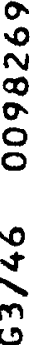

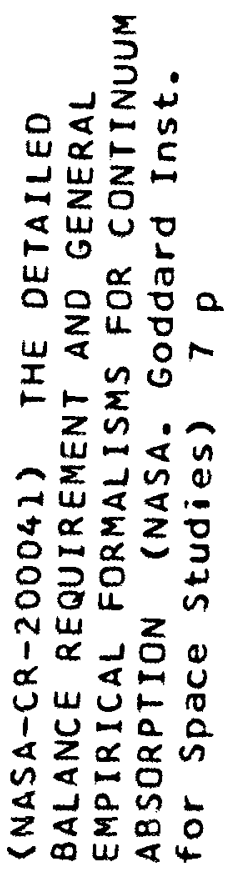

\section{INTRODUCTION}

Transmission codes ${ }^{1-6}$ which calculate atmospheric transmittance and radiance for various geometries and atmospheric conditions are widely used in remote sensing, communication, etc. It is essential for the further development and refinement of these transmission codes to take into account the continuum absorption by $\mathrm{H}_{2} \mathrm{O}-\mathrm{H}_{2} \mathrm{O}$ and $\mathrm{H}_{2} \mathrm{O}-\mathrm{N}_{2}$ pairs in the window regions of the Earth's atmosphere. Although a lot of effort has been made for decades, both experimentally and theoretically, ${ }^{7-12}$ we have not yet achieved this goal. However, it has been shown that the results of line-by-line calculations using the Lorentz line shape, which is valid near line centers, cannot be used to explain the continuum absorption; this implies that the Lorentz line shape must be modified in the wings of the lines.

Recent theoretical studies ${ }^{13-21}$ on continuum absorption have led to significant progress in our understanding of the physical mechanisms and enable us to predict its magnitude and its temperature dependence. These studies complement existing empirical models especially at low temperatures at which adequate experimental measurements are lacking, however insufficient the present accuracy may be for atmospheric applications. On the other hand, empirically modeling the continuum absorption by introducing an adjustable parameter, a $\chi$ factor, ${ }^{22}$ to modify the commonly used Lorentz line shape provides a simple methodology for practical applications and it is widely used in transmission codes. ${ }^{1-6}$ Of course, the reliability of the empirical models is limited by various other factors. One of them stems from uncertainties in the available experimental data on which the empirical models are based. Another is the limited temperature range and frequency regions covered by the reported measurements. In addition, the form of the empirical line shape correction affects its accuracy in representing the continuum absorption. Unfortunately, the empirical model ${ }^{23}$ that is widely used in transimission codes fails to satisfy the principle of detailed balance which governs the emission and absorption processes. We focus our attention on the detailed balance requirement in the present paper and show how the empirical model can be improved.

In Secs. 2.1 and 2.2 we briefly outline the basic formulae for the correlation function and the spectral density and give explicit expressions involving the Lorentz line shape. We analyse the spectral density obtained with a $\chi$ factor modifying the Lorentz line shape and identify in Sec. 2.3 the source of its failure to satisfy the detailed balance requirement. Then, in Sec. 2.4, a simple 
improved empirical model which satisfies detailed balance is presented. We discuss in Sec. 2.5 a more general empirical model in which not only the detailed balance principle, but also the asymmetric character of line shape has been taken into account. Finally, some conclusions are drawn in Sec. 3.

\section{THE GENERAL FORMALISM}

\section{I. The correlation function and spectral density}

As is well known, the absorption of radiation at frequency $\omega\left(\mathrm{cm}^{-1}\right)$ by a gaseous sample in thermal equilibrium at temperature $T$ is characterized by the absorption coefficient $\alpha(\omega)$

$$
\alpha(\omega)=\frac{4 \pi^{2}}{3 \hbar c} \omega t h(h \omega / 2 k T) \frac{1}{2 \pi} \int_{-\infty}^{\infty} e^{-\omega \omega}\left[\left\langle\mu^{(H)}(0) \cdot \mu^{(H)}(t)\right\rangle+\left\langle\mu^{(H)}(t) \cdot \mu^{(H)}(0)\right\rangle\right] \mathrm{d} t .
$$

In this expression, $\mu^{(\mathcal{H})}(t)$ is the total dipole operator of the gas in the Heisenberg representation

$$
\mu^{(H)}(t)=\mathrm{e}^{i H / \hbar / \mu} \mu^{(H)}(0) \mathrm{e}^{-\mathrm{H} t / \hbar},
$$

and the angular brackets denote the ensemble average (i.e., the correlation function)

$$
\left\langle\mu^{(H)}(0) \cdot \mu^{(H)}(t)\right\rangle=\sum_{i} \rho_{i}\left\langle i\left|\mu^{(H)}(0) \cdot \mu^{(H)}(t)\right| i\right\rangle,
$$

where $\rho_{i}$ is the normalized Boltzmann factor of the initial state $i$. The spectral density $F(\omega)$ is a Fourier transform of the correlation function defined by

$$
F(\omega) \equiv \frac{1}{2 \pi} \int_{-\infty}^{\infty} \mathrm{e}^{-i \omega t}\langle\mu(0) \cdot \mu(t)\rangle \mathrm{d} t,
$$

and $F(-\omega)$ is defined by

$$
F(-\omega) \equiv \frac{1}{2 \pi} \int_{-\infty}^{\infty} \mathrm{e}^{-i \omega t}\langle\mu(t) \cdot \mu(0)\rangle \mathrm{d} t .
$$

For simplicity in the above expressions we have dropped the Heisenberg superscript. The spectral density must satisfy the detailed balance requirement

$$
F(-\omega)=\mathrm{e}^{-\omega_{0} / k t} F(\omega)
$$

We note that the definition of the Fourier transform in Eqs. (4) and (5) is the same as that used to derive the empirical model $\left.\right|^{22.23}$ but differs from that used in our previous papers ${ }^{17-21}$ by a minus sign in the exponential.

\subsection{The correlation function and the spectral density corresponding to the Lorentz line shape}

Within the impact approximation the correlation functions are given by

$$
\begin{aligned}
& \langle\mu(0) \cdot \mu(t)\rangle=\sum_{i f} \rho_{i}\left|\mu_{f}\right|^{2} e^{-\gamma_{\mu} \mid \omega+\omega_{s}}, \\
& \langle\mu(t) \cdot \mu(0)\rangle=\sum_{f} \ddot{\rho_{i}}\left|\ddot{\mu}_{f}\right|^{2} \mathrm{e}^{-r_{f} \mid t-\log _{f} t}=\langle\mu(0) \cdot \mu(t)\rangle^{*},
\end{aligned}
$$

where $\omega_{f i}=-\omega_{i j}, \gamma_{f i}=\gamma_{i f}$ and $\left|\mu_{f}\right|^{2}=\left|\mu_{i j}\right|^{2}$, and the spectral density is given by

$$
\begin{aligned}
F(\omega) & =\frac{1}{2 \pi} \sum_{i f} \rho_{i}\left|\mu_{f}\right|^{2} \int_{-\infty}^{\infty} e^{\left.-\alpha \omega-\omega_{j}\right) \gamma-\nu_{j} \mid \pi} \mathrm{d} t \\
& =\frac{1}{\pi} \sum_{i f} \rho_{i}\left|\mu_{f i}\right|^{2} \frac{\gamma_{j i}}{\left(\omega-\omega_{f}\right)^{2}+\gamma_{f}^{2}},
\end{aligned}
$$


and

$$
\begin{aligned}
F(-\omega) & =\frac{1}{2 \pi} \sum_{i f} \rho_{i}\left|\mu_{f}\right|^{2} \int_{-\infty}^{\infty} \mathrm{e}^{-i\left(\omega+\omega_{f}\right) \nu-y_{j} \mid n} \mathrm{~d} t \\
& =\frac{1}{\pi} \sum_{i f} \rho_{i}\left|\mu_{f}\right|^{2} \frac{\gamma_{f}}{\left(\omega+\omega_{f}\right)^{2}+\gamma_{f}^{2}} \\
& =\mathrm{e}^{-k \omega / k T} \frac{1}{\pi} \sum_{i f} \mathrm{e}^{m\left(\omega-\omega_{f}\right) / k T} \rho_{i}\left|\mu_{f}\right|^{2} \frac{\gamma_{f}}{\left(\omega-\omega_{f}\right)^{2}+\gamma_{f}^{2}} .
\end{aligned}
$$

The fact that the line shapes obtained based on the impact approximation are Lorentzian is clearly shown in Eqs. (9) and (10). The spectral density given above does not satisfy the detailed balance requirement. However, in the band center region where most of the contributions come from nearby strong lines and where the Lorentz line shape is valid, this requirement is approximately satisfied. The symmetric spectral density can be expressed as

$$
\begin{aligned}
F(\omega)+F(-\omega) & =\frac{3 h c}{4 \pi^{2}} \frac{1}{\pi} \sum_{\omega_{f}>0} \frac{S_{f}}{\omega_{f} t h\left(h \omega_{f} / 2 k T\right)}\left[\frac{\gamma_{f}}{\left(\omega-\omega_{f i}\right)^{2}+\gamma_{f}^{2}}+\frac{\gamma_{f}}{\left(\omega+\omega_{f}\right)^{2}+\gamma_{f}^{2}}\right] \\
& =\frac{3 h c}{4 \pi^{2}} \frac{1}{\pi} \sum_{\omega_{j}>0} S_{f_{f}}\left[\frac{\gamma_{f}}{\left(\omega-\omega_{f}\right)^{2}+\gamma_{f}^{2}}+\frac{\gamma_{f}}{\left(\omega+\omega_{f}\right)^{2}+\gamma_{f}^{2}}\right] .
\end{aligned}
$$

where the usual line strength $S_{f}$ is defined by

$$
S_{f}=\frac{4 \pi^{2}}{3 \hbar c} \omega_{f i}\left|\mu_{f}\right|^{2}\left(\rho_{i}-\rho_{f}\right)
$$

and a reduced line strength $\tilde{S}_{f}$ is defined by

$$
\bar{S}_{f} \equiv \frac{S_{f}}{\omega_{f} t h\left(\hbar \omega_{f} / 2 k T\right)} .
$$

\subsection{A widely used empirical model of the spectral density for the continuum absorption}

It is well known that the Lorentz line shape is not valid in the line-wing region. In order to take into account the derivations from the Lorentz line shape, an empirical $\chi(\omega)$ factor has been introduced ${ }^{23}$ in the expression of the spectral density, Eqs. (9) and (10), and it has been arbitrarily assumed to be an even function,

$$
\begin{gathered}
F(\omega)=\frac{1}{\pi} \sum_{i f} \rho_{i}\left|\mu_{f}\right|^{2} \frac{\gamma_{f}}{\left(\omega-\omega_{f}\right)^{2}+\gamma_{f}^{2}} \chi\left(\omega-\omega_{f}\right), \\
F(-\omega)=\frac{1}{\pi} \sum_{i f} \rho_{i}\left|\mu_{f}\right|^{2} \frac{\gamma_{f}}{\left(\omega+\omega_{f}\right)^{2}+\gamma_{f}^{2}} \chi\left(\omega+\omega_{f}\right) .
\end{gathered}
$$

Then, the symmetric spectral density is given by

$$
\begin{aligned}
F(\omega)+F(-\omega)= & \frac{3 \hbar c}{4 \pi^{2}} \frac{1}{\pi} \sum_{\omega_{h}>0} \frac{S_{f}}{\omega_{f} \ln \left(\hbar \omega_{f} / 2 k T\right)}\left[\frac{\gamma_{f}}{\left(\omega-\omega_{f}\right)^{2}+\gamma_{f i}^{2}} \times\left(\omega-\omega_{f}\right)\right. \\
& \left.+\frac{\gamma_{f}}{\left(\omega+\omega_{f}\right)^{2}+\gamma_{f}^{2}} \times\left(\omega+\omega_{f i}\right)\right] \\
= & \frac{3 h c}{4 \pi^{2}} \frac{1}{\pi} \sum_{\omega_{h}>0} S_{f}\left[\frac{\gamma_{f}}{\left(\omega-\omega_{f}\right)^{2}+\gamma_{f}^{2}} \times\left(\omega-\omega_{f i}\right)\right. \\
& \left.+\frac{\gamma_{f}}{\left(\omega+\omega_{f}\right)^{2}+\gamma_{f i}^{2}} \times\left(\omega+\omega_{f i}\right)\right] .
\end{aligned}
$$

One expects that the empirical factor $\chi(\omega)$ approaches unity as $\omega \rightarrow 0$ and falls off very quickly after $\omega$ is beyond a certain region. By fitting the experimental data with the expression Eq. (16), one is able to obtain an explicit form of the $\chi$ function. ${ }^{23}$ Unfortunately, the spectral density given above does not satisfy the detailed balance requirement due to the assumption made on the evenness of the $\chi$ factor. 


\subsection{A modified empirical expression for the spectral density}

One can obtain a modified empirical model by introducing a $\chi$ factor in the expressions of the spectral densities

$$
\begin{gathered}
F(\omega)=\frac{1}{\pi} \sum_{i f} \rho_{i}\left|\mu_{f}\right|^{2} \frac{y_{f i}}{\left(\omega-\omega_{f}\right)^{2}+\gamma_{f}^{2}} \chi\left(\omega-\omega_{f}\right), \\
F(-\omega)=\frac{1}{\pi} \sum_{i j} \rho_{i}\left|\mu_{f i}\right|^{2} \frac{\gamma_{f}}{\left(\omega+\omega_{f}\right)^{2}+\gamma_{f}^{2}} x\left(-\omega-\omega_{f}\right) .
\end{gathered}
$$

However, instead of assuming that the $\chi$ factor introduced above is an even function as done previously, one imposes the requirement of detailed balance on the spectral density:

$$
\begin{aligned}
F(-\omega) & =\mathrm{e}^{-\kappa_{\omega} / k T} F(\omega) \\
& =\mathrm{e}^{-\lambda_{\omega} / k T} \frac{1}{\pi} \sum_{i f} \rho_{i}\left|\mu_{f}\right|^{2} \frac{\gamma_{f}}{\left(\omega-\omega_{f}\right)^{2}+\gamma_{f}^{2}} \chi\left(\omega-\omega_{f}\right) \\
& =\mathrm{e}^{-\lambda_{\omega} / k T} \frac{1}{\pi} \sum_{i f} \rho_{f}\left|\mu_{f}\right|^{2} \frac{\gamma_{f}}{\left(\omega+\omega_{f}\right)^{2}+\gamma_{f}^{2}} \times\left(\omega+\omega_{f}\right) .
\end{aligned}
$$

Comparing the corresponding resonant terms in expressions (18) and (19), the following equality must hold

$$
\chi\left(-\omega-\omega_{f}\right)=e^{-m\left(\omega+\omega_{f}\right) / k T} \chi\left(\omega+\omega_{f}\right) .
$$

The conclusion is that in order to satisfy the detailed balance requirement, the empirical $\chi$ factor should not be an even function, but

$$
\chi(-\omega)=e^{-\kappa \omega \mid k T} \chi(\omega)
$$

or expressed more symmetrically,

$$
e^{\Lambda \omega / 2 k T} \chi(-\omega)=e^{-\kappa_{\omega} / 2 k T} \chi(\omega) .
$$

Therefore, it is the symmetric factor, $\hat{\chi}(\omega)$, defined by

$$
\hat{\chi}(\omega) \equiv \mathrm{e}^{-\kappa \omega / 2 k T} \chi(\omega),
$$

and not $\chi(\omega)$ that is an even function; i.e.

$$
\hat{\chi}(\omega)=\hat{\chi}(-\omega) \text {. }
$$

In terms of $\hat{\chi}$ the modified spectral density can be expressed as

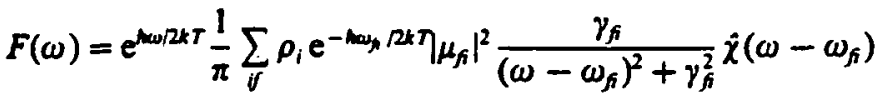

$$
\begin{aligned}
& =e^{\kappa \omega / 2 k T} \frac{1}{\pi} \sum_{i f}\left\|\mu_{f}\right\|^{2} \frac{\gamma_{f}}{\left(\omega-\omega_{f}\right)^{2}+\gamma_{f}^{2}} \hat{\chi}\left(\omega-\omega_{f}\right),
\end{aligned}
$$

where the symmetric line intensities $\left\|\mu_{f}\right\|^{2}\left(=\left\|\mu_{i j}\right\|^{2}\right)$ are defined by

$$
\left\|\mu_{f}\right\|^{2} \equiv\left(\rho_{i} \rho_{f}\right)^{12}\left|\mu_{s}\right|^{2}=g_{f} e^{-m E_{f}+\varepsilon_{i} / 2 k T}\left|\mu_{f}\right|^{2} / Q
$$

in which $g\left(=g_{i}\right)$ is the nuclear spin degeneracy factor and $Q$ is the partition function. Similarly, using Eqs. (19), (22), (23) and (25), we have

$$
F(-\omega)=\mathrm{e}^{-\omega_{\omega} / 2 \pi T} \frac{1}{\pi} \sum_{i f}\left\|\mu_{f}\right\|^{2} \frac{\gamma_{f}}{\left(\omega-\omega_{f}\right)^{2}+\gamma_{f}^{2}} \hat{x}\left(\omega-\omega_{f}\right) . \therefore
$$

More clearly, if we define $G(\omega)$ by

$$
G(\omega) \equiv \frac{1}{\pi} \sum_{i j}\left\|\mu_{f}\right\|^{2} \frac{\gamma_{f}}{\left(\omega-\omega_{f}\right)^{2}+\gamma_{f}^{2}} \hat{\chi}\left(\omega-\omega_{f}\right),
$$


which is an even function, then the spectral density can be simply expressed as

$$
\begin{aligned}
& F(\omega)=\mathrm{e}^{\mu / 2 \mu T} G(\omega), \\
& F(-\omega)=e^{-k_{0} / 2 t} G(\omega) .
\end{aligned}
$$

Therefore, this modified spectral density does satisfy the detailed balance requirement which can be expressed more symmetrically as

$$
e^{-n_{0} / 2 k T} F(\omega)=e^{\kappa_{0} / 2 k T} F(-\omega) \text {. }
$$

Finally, the symmetric modified spectral density is

$$
\begin{aligned}
F(\omega)+F(-\omega)= & 2 \operatorname{ch}(h \omega / 2 k T) G(\omega)=\frac{3 h c}{4 \pi^{2}} \frac{1}{\pi} \sum_{\omega_{f}>0} S_{f} \frac{c h(h \omega / 2 k T)}{c h\left(h \omega_{f} / 2 k T\right)}\left[\frac{\gamma_{f}}{\left(\omega-\omega_{f}\right)^{2}+\gamma_{f}^{2}} \hat{\chi}\left(\omega-\omega_{f}\right)\right. \\
& \left.+\frac{\gamma_{f}}{\left(\omega+\omega_{f}\right)^{2}+\gamma_{f i}^{2}} \hat{\chi}\left(\omega+\omega_{f i}\right)\right] .
\end{aligned}
$$

We note that the modified empirical expression of the spectral density has the same form as that derived by Rosenkranz in his theoretical formalism. ${ }^{13.14}$

By comparing the above expression with the corresponding expression (16) in which both of the empirical factors denoted by $\hat{\chi}(\omega)$ and $\chi(\omega)$, respectively, are even functions, one is able to find that these two expressions differ from each other by the factor $\operatorname{ch}(h \omega / 2 k T) / \operatorname{ch}\left(h \omega_{f} / 2 k T\right)$. It is obvious that in the band center regions where most of the contributions to absorption come from nearby strong resonant lines and where $\omega-\omega_{f} \approx 0$, the difference between these two formulas can be ignored. However, since atmospheric windows are the primary regions in which the empirical factors play a crucial role, including or excluding the factor $\operatorname{ch}(h \omega / 2 k T) / \operatorname{ch}\left(\hbar \omega_{f} / 2 k T\right)$ makes significant differences. In addition, these differences will increase as the temperature decreases. Although the results of continuum absorption obtained from these two formulae can be made to coincide in a given frequency region for a specified temperature (for example, in the $10 \mu \mathrm{m}$ window at room temperature for which experimental data are available and on which the empirical fittings are partially based), differences will definitely appear when predicting the absorption by interpolation or extrapolation to other temperatures and/or frequency regions; sample numerical tests confirm this conclusion. Therefore, the intrinsic weakness of Eq. (16) will limit its reliability for atmospheric applications.

\subsection{A more general modified spectral density}

More generally, in order to distinguish the positive-frequency resonance and the negative-frequency resonance, one has to introduce two $\chi$ factors, $x+$ and $x$-instead of one $\chi$ factor in the empirical model, ${ }^{19-21}$

$$
\begin{aligned}
& F(\omega)=\frac{1}{\pi} \sum_{\omega_{f}>0}\left|\mu_{f}\right|^{2}\left[\rho_{i} \frac{\gamma_{f}}{\left(\omega-\omega_{f}\right)^{2}+\gamma_{f}^{2}} \chi_{+}\left(\omega-\omega_{f}\right)+\rho_{f} \frac{\gamma_{f}}{\left(\omega+\omega_{f}\right)^{2}+\gamma_{f}^{2}} \chi_{-}\left(\omega+\omega_{f}\right)\right], \\
& F(-\omega)=\frac{1}{\pi} \sum_{\omega_{f}>0}\left|\mu_{f i}\right|^{2}\left[\rho_{i} \frac{\gamma_{f}}{\left(\omega+\omega_{f}\right)^{2}+\gamma_{f}^{2}} \chi_{+}\left(-\omega-\omega_{f}\right)+\rho_{f} \frac{\gamma_{f}}{\left(\omega-\omega_{f}\right)^{2}+\gamma_{f}^{2}} x_{-}\left(-\omega+\omega_{f}\right)\right] .
\end{aligned}
$$

Again, imposing the requirement of the detailed balance, $F(-\omega)=e^{-\omega_{0} / k T} F(\omega)$, on the spectral density, we must have,

$$
\therefore \quad \begin{aligned}
& e^{N \omega / 2 k T} X_{+}(-\omega)=e^{-k_{0} / 2 k T} X_{-}(\omega), \\
& e^{N_{0} / 2 k T} x_{-}(-\omega)=e^{-\kappa_{0} / 2 k T} X_{+}(\omega) .
\end{aligned}
$$

Similarly, we can define

$$
\begin{aligned}
& \hat{\chi}_{+}(\omega) \equiv e^{-\omega_{0} / 2 k T} \chi_{+}(\omega), \\
& \hat{\chi}_{-}(\omega) \equiv e^{-\omega_{0} / 2 k T} \chi_{-}(\omega) .
\end{aligned}
$$


We note that neither of these two factors is an even function, rather they are related to each other by an inversion operator $I$,

$$
\begin{aligned}
& \hat{\chi}_{+}(\omega)=I \hat{\chi}_{-}(\omega) \equiv \hat{\chi}_{-}(-\omega), \\
& \hat{\chi}_{-}(\omega)=I \hat{\chi}_{+}(\omega) \equiv \hat{\chi}_{+}(-\omega) .
\end{aligned}
$$

In terms of them, the modified spectral density can be expressed as

$$
F(\omega)=\mathrm{e}^{k^{2} / 2 k T} \frac{1}{\pi} \sum_{\omega_{f}>0}\left\|\mu_{f}\right\|^{2}\left[\frac{\gamma_{f i}}{\left(\omega-\omega_{f}\right)^{2}+\gamma_{f i}^{2}} \hat{x}_{+}\left(\omega-\omega_{f}\right)+\frac{\gamma_{f}}{\left(\omega+\omega_{f}\right)^{2}+\gamma_{f}^{2}} \hat{x}_{-}\left(\omega+\omega_{f}\right)\right],
$$

and

$$
F(-\omega)=\mathrm{e}^{-k \omega / 2 k T} \frac{1}{\pi} \sum_{\omega_{f}>0}\left\|\mu_{f_{f}}\right\|^{2}\left[\frac{\gamma_{f}}{\left(\omega+\omega_{f}\right)^{2}+\gamma_{f}^{2}} \hat{\chi}_{-}\left(\omega+\omega_{f}\right)+\frac{\gamma_{f}}{\left(\omega-\omega_{f}\right)^{2}+\gamma_{f}^{2}} \hat{\chi}_{+}\left(\omega-\omega_{f}\right)\right] .
$$

More clearly, if we define

$$
G^{\prime}(\omega) \equiv \frac{1}{\pi} \cdot \sum_{\omega_{f}>0}\left\|\mu_{f}\right\|^{2}\left[\frac{\gamma_{f}}{\left(\omega-\omega_{f}\right)^{2}+\gamma_{f}^{2}} \hat{\chi}_{+}\left(\omega-\omega_{f}\right)+\frac{\gamma_{f}}{\left(\omega+\omega_{f}\right)^{2}+\gamma_{f}^{2}} \hat{\chi}_{-}\left(\omega+\omega_{f}\right)\right]
$$

which is an even function, then we have

$$
\begin{gathered}
F(\omega)=\mathrm{e}^{\kappa \omega / 2 k T} G^{\prime}(\omega), \\
F(-\omega)=\mathrm{e}^{-\hbar \omega / 2 k T} G^{\prime}(\omega),
\end{gathered}
$$

where the fact that the detailed balance requirement is satisfied is clearly shown. The symmetric modified spectral density can accordingly be expressed as

$$
\begin{aligned}
F(\omega)+F(-\omega)= & 2 \operatorname{ch}(h \omega / 2 k T) G^{\prime}(\omega) \\
= & \frac{3 \hbar c}{4 \pi^{2}} \frac{1}{\pi} \sum_{\omega_{f}>0} \tilde{S}_{f} \frac{c h(h \omega / 2 k T)}{c h\left(h \omega_{f} / 2 k T\right)}\left[\frac{\gamma_{f}}{\left(\omega-\omega_{f}\right)^{2}+\gamma_{f}^{2}} \hat{\chi}_{+}\left(\omega-\omega_{f}\right)\right. \\
& \left.+\frac{\gamma_{f}}{\left(\omega+\omega_{f}\right)^{2}+\gamma_{f}^{2}} \hat{\chi}_{-}\left(\omega+\omega_{f}\right)\right] .
\end{aligned}
$$

By introducing two $\chi$ factors, $\hat{\chi}_{+}(\omega)$ and $\hat{\chi}_{-}(\omega)$, in the above expression, the asymmetric character of the line shapes is explicitly formulated. However, if we do not distinguish between $\hat{\chi}_{+}$and $\hat{\chi}_{-}$ and replace both of them by the same $\hat{\chi}$, Eq. (40) will be the same as Eq. (30). We note that the expression of spectral density in terms of two $\chi$ factors is not only more general than the expression in terms of one $\chi$ factor, but also has a sound physical basis. ${ }^{\mid 9-21}$

\section{CONCLUSIONS}

As has been pointed out in Sec. 2.3 , the empirical model widely used in transmission codes fails to satisfy the detailed balance requirement. Its failure can be traced back to an arbitrary assumption that the factor $\chi(\omega)$ introduced to modify the Lorentz line shape is an even function. By lifting this assumption and introducing the symmetric empirical function $\hat{\chi}(\omega)$ instead of $\chi(\omega)$, one is able to obtain an empirical formalism which satisfies the detailed balance requirement and overcomes the intrinsic weakness of the previous formalism. In this formalism, it is $\hat{\chi}(\omega)$, not $\chi(\omega)$ itself, that is an even function. In addition, in order to derive a more general expression of the spectral density, one has to distinguish the positive-resonance terms from the negative-reson. ance terms and, consequently, none of the $\chi$ factors is an even function. Therefore, one has to introduce two $\chi$ factors, one for the positive frequency region and the other for the negative frequency region, ${ }^{19-21}$ in order to take into account the asymmetric character of the line shapes. This more general empirical formalism also satisfies the detailed balance requirement and has a more sound physical basis. This latter formalism should be used in the further development of transmission codes. While this paper has focused on the spectral densities, a parallel study on their time domain partners, i.e., the correlation functions has also been performed and will be presented elsewhere. 
Acknowledgements-This work was supported in part by the Department of Energy Interagency Agreement under the Atmospheric Radiation Measurement Program and in part by NASA grant No. NAGW-3785. We would like to thank S. A. Clough for helpful discussions. One author (Q. Ma) would like to thank B. Carlson and S. Green for reading and improving the manuscript.

\section{REFERENCES}

I. F. X. Kneizys, E. P. Shettle, W. O. Gallery, J. H. Chetwynd, L. W. Abreu, J. E. A. Selby, R. W. Fenn, and R. A. McClatchey, Report AFGL-TR-80-0067 (1980).

2. F. X. Kneizys, E. P. Shettle, W. O. Gallery, J. H. Chetwynd, L. W. Abreu, J. E. A. Selby, S. A. Clough, and R. W. Fenn, Report AFGL-TR-83-0187 (1983).

3. S. A. Clough, F. X. Kneizys, E. P. Shettle, and G. P. Anderson, Proc. Geh Conf. on Atmospheric Radiation, AMS, Boston, MA, 141 (1986).

4. F. X. Kneizys, E. P. Shettle, L. W. Abreu, G. P. Anderson, J. H. Chetwynd, W. O. Gallery, J. E. A. Selby, and S. A. Clough, Report AFGL-TR-88-0177 (1988).

S. A. Berk, L. S. Bernstein, and D. C. Robertson, Report GL-TR-89-0122 (1989).

6. R. G. Isaacs, S. A. Clough, R. D. Worsham, J. L. Moncet, B. L. Lindner, and L. D. Kaplan, Report GL-TR-90-0080 (1990).

7. Atmospheric Water Vapor, A. Deepak, T. D. Wilkerson, and L. H. Runke, eds., Academic Press, New York, NY (1980).

8. D. E. Burch, SPIE Proc. 277, 28 (1981); D. E. Burch and D. A. Gryvnak, Report AFGL-TR-79-0054 (1979); D. E. Burch and R. L. Alt, Report AFGL-TR-84-0128 (1984).

9. P. Varanasi, SPIE Proc. 928, 213 (1988).

10. W. B. Grant, Appl. Opt. 29, 451 (1990).

11. M. E. Thomas, Infrared Phys. 30, 161 (1990).

12. R. W. Davies, R. H. Tipping, and S. A. Clough, Phys. Rev. A 26, 3378 (1982).

13. P. W. Rosenkranz, J. Chem. Phys. 83, 6139 (1985).

14. P. W. Rosenkranz, J. Chem. Phys. 87, 163 (1987).

15. C. Boulet, J. Boissoles, and D. Robert, J. Chem. Phys. 89, 625 (1988).

16. J. Boissoles, V. Menoux, R. Le Doucen, C. Boulet, and D. Robert, J. Chem. Phys. 91, 2163 (1989).

17. Q. Ma and R. H. Tipping, J. Chem. Phys. 93, 7066 (1990).

18. Q. Ma and R. H. Tipping, J. Chem. Phys. 93, 6127 (1990).

19. Q. Ma and R. H. Tipping, J. Chem. Phys. 95, 6290 (1991).

20. Q. Ma and R. H. Tipping, J. Chem. Phys. 96, 8655 (1992).

21. Q. Ma and R. H. Tipping, J. Chem. Phys. 97, 818 (1992).

22. S. A. Clough, F. X. Kneizys, R. W. Davies, R. Gamache, and R. H. Tipping, in Atmospheric Water Vapor, A. Deepak, T. D. Wilkerson, and L. H. Runke, eds., Academic Press, New York, NY (1980).

23. S. A. Clough, F. X. Kneizys, and R. W. Davies, Atmos. Res. 23, 229 (1989). 